


\title{
Caracterización del lenguaje en las variantes lingüísticas de la Afasia Progresiva Primaria
}

\author{
Characterization of language in the linguistic variants of \\ Primary Progressive Aphasia
}

Ma Cruz Pérez Lancho

UNIVERSIDAD PONTIFICIA DE SALAMANCA

ESPAÑA

mcperezla@upsa.es

\author{
Sergio García Bercianos \\ Centro Sanitario PSicológico SELEMARK \\ ESPAÑA \\ sergiogarciacorreo@gmail.com
}

Recibido: 30-XI-2018 / Aceptado: 03-VII-2019

DOI: $10.4067 /$ S0718-09342020000100198

\section{Resumen}

La Afasia Progresiva Primaria (APP) incluye a un conjunto de trastornos neurocognitivos con una afectación principal de la función lingüística. Se caracteriza por la preservación inicial del funcionamiento en las otras áreas cognitivas, mientras se va deteriorando la autonomía del individuo de forma significativa (Mesulam, 2016). Los recientes avances en neuroimagen y hallazgo de biomarcadores han contribuido a un mejor conocimiento de estos síndromes, aunque han generado a su vez la necesidad de revisar los criterios diagnósticos y la configuración clínica de sus variantes, con un resultado no exento de controversias. El objetivo de este trabajo es describir los síntomas del lenguaje que caracterizan a los distintos subtipos de APP, describiendo los criterios actualizados para su diagnóstico y las recientes clasificaciones de este grupo de patologías. En primer lugar, se presenta la 'APP-variante no fluente' o 'agramática', caracterizada por anormalidades en un tipo de discurso que resulta forzado, lento y telegráfico, concomitante con apraxia del habla o disartria. En segundo lugar, se describe la 'APP-variante semántica', caracterizada por un discurso fluido pero vacío de contenido, con uso de palabras genéricas y una relativa anomia. Por último, se analiza la 'APP-variante logopénica', caracterizada por la presencia de frecuentes pausas en el discurso y circunloquios originados por la dificultad mnésica para encontrar las palabras, con una relativa conservación de la gramática, en un perfil de deterioro lingüístico muy similar al de la Enfermedad de Alzheimer.

Palabras Clave: Demencia frontotemporal, alteraciones del lenguaje, APP-variante agramática, APP-variante semántica, APP-variante logopénica. 


\begin{abstract}
Primary Progressive Aphasia (PPA) includes a set of neurocognitive disorders with a major impairment in the linguistic function. It is characterized by the initial preservation of functioning in the other cognitive areas, while the autonomy of the individual deteriorates significantly (Mesulam, 2016). Recent advances in neuroimaging and finding of biomarkers have contributed to a better knowledge of these syndromes, although they have generated in turn the need to review the diagnostic criteria and the clinical configuration of their variants, with a result not without controversy. The objective of this work is to describe the language symptoms that characterize the different subtypes of PPA, describing the updated criteria for its diagnosis and the recent classifications of this group of pathologies. First, the 'non-fluent or agrammatic PPA-variant' is presented, characterized by abnormalities in a type of speech that is forced, slow and telegraphic, concomitant with speech apraxia or dysarthria. Secondly, the 'PPA-semantic variant' is described, characterized by a fluid speech, but empty of content, with the use of generic words and a relative anomie. Finally, we analyze the 'PPA-logopenic variant', characterized by the presence of frequent pauses in the discourse and circumlocutions caused by the mnesic difficulty to find the words, with a relative conservation of the grammar, in a profile of linguistic deterioration very similar to that of Alzheimer's Disease.
\end{abstract}

Key Words: Frontotemporal dementia, language alterations, APP agrammatic variant, APP semantic variant, APP logopenic variant.

\title{
INTRODUCCIÓN
}

Las 'Demencias lobares frontotemporales' constituyen un grupo heterogéneo de enfermedades neurodegenerativas con diferentes histopatologías, expresiones genéticas y sintomatología clínica, caracterizado por una afectación progresiva de los lóbulos frontales y temporales y asociado a pérdida neuronal y gliosis. Se las considera la tercera causa de demencia, después de la enfermedad de Alzheimer (EA) y la demencia por cuerpos de Lewy (Toribio-Díaz \& Morera-Guitart, 2008). Tienen una prevalencia estimada de 15-22 casos/100.000 habitantes y son las demencias más comunes en adultos menores de 65 años, siendo la edad de presentación más frecuente entre los 50-60 años (Ratnavalli, Brayne, Dawson \& Hodges, 2002).

El término 'Demencia frontotemporal' (DFT) en la actualidad comprendería tres sub-síndromes: uno caracterizado por el deterioro progresivo del área conductual y del control ejecutivo y dos con una afectación fundamental en el dominio del lenguaje. Además, recientemente se ha descrito una tercera variante lingüística relacionada con la enfermedad de Alzheimer (Woollacott \& Rohrer, 2016) cuyas características resultan limítrofes entre ambos trastornos neurocognitivos. Todas las variantes lingüísticas de la DFT se agrupan bajo el término de 'Afasia progresiva primaria' (APP), utilizado para describir un síndrome clínico que se manifiesta por un deterioro diferencial del área del lenguaje respecto al resto de las funciones cognitivas superiores 
y que debe ser causado por una enfermedad degenerativa del tejido cerebral (Mesulam, 2016).

Desde su primera descripción clínico-patológica, hace más de un siglo, ha sido creciente el interés de esta patología gracias a los avances en las técnicas de neuroimagen y el hallazgo de biomarcadores. Sin embargo, estas aportaciones han obligado a la comunidad científica a reconceptualizar y rediseñar los criterios diagnósticos de estas enfermedades, en un intento de integrar los aspectos neuropatológicos con las observaciones clínicas. En la actualidad, los esfuerzos se centran en aunar las aportaciones de diversos ámbitos de estudio para buscar correlaciones entre la expresión de la enfermedad a nivel neuronal, las áreas cerebrales y su conectividad, tanto estructural como funcional, las proteinopatías subyacentes, los estudios genéticos y de neuroimagen con los fenotipos o síndromes clínicos (Pievani, Filippini, Van Den Heuvel, Cappa \& Frisoni, 2014).

Como respuesta a estos desafíos, en los últimos años se han propuesto sucesivos criterios para diagnosticar y clasificar este grupo de condiciones con el fin de presentar una terminología común que permitan rotular casos y compartir información clínica (Lillo \& Leyton, 2016). Sin embargo, la coexistencia de esta diversidad de clasificaciones ha originado cierta confusión en la práctica clínica que debe ser detectada y clarificada (Pérez-Lancho \& García-Bercianos, 2018).

Por este motivo, la lingüística clínica se enfrenta también al reto de actualizar la caracterización de las alteraciones del lenguaje en cada una de las variantes de la APP a la luz de las nuevas incorporaciones diagnósticas. El objetivo de este trabajo es describir la sintomatología lingüística de los distintos subtipos de APP, partiendo de los criterios actualizados para su diagnóstico y las recientes clasificaciones de este grupo de patologías.

Para ello hemos realizado una revisión bibliográfica de la literatura científica sobre APP en Pubmed y Google Académico, con las palabras clave APP, DFT y lenguaje, desde el año 2000, en castellano y en inglés. Fueron considerados 248 trabajos, de los cuales se excluyeron 207 y se seleccionaron 41. Se analizaron los artículos relacionados con la evolución conceptual de la APP, sus criterios diagnósticos y caracterización lingüística. También se revisaron algunos importantes artículos con estudios de neuroimagen que presentaban correspondencias anátomo-funcionales de las alteraciones del lenguaje. Además, se añadieron seis trabajos anteriores al año 2000 considerando su relevancia histórica.

Nuestro trabajo describe el surgimiento de los distintos criterios diagnósticos desde una perspectiva histórica y presenta los rasgos clínicos del lenguaje y los correlatos anatómicos de sus tres grandes formas clínicas. En primer lugar, de la 'APP-variante no fluente' o 'agramática', de discurso lento y telegráfico, asociado a apraxia del habla o disartria. En segundo lugar, de la 'APP-variante semántica', de discurso fluente pero 
vacío de contenido, por pérdida de la asociación entre la palabra y su significado. Por último, de la 'APP-variante logopénica', de discurso gramaticalmente conservado, pero con abundantes circunloquios y pausas de carácter amnésico, en un perfil de deterioro análogo al de la EA.

\section{Evolución conceptual de las Afasias Progresivas Primarias}

Se atribuye a Pick (1904) la descripción de los primeros casos de demencia por atrofia en el lóbulo temporal izquierdo, con hallazgos de cuerpos de Pick en el tejido neuronal y no debidos a una lesión cerebrovascular. Estos estudios describían la aparición de alteraciones progresivas tanto de la conducta como del lenguaje de los pacientes (Mesulam, Rogalski, Wieneke, Hurley, Geula, Bigio, Thompson \& Weintraub, 2014). Años después, en 1906, Alzheimer (1911) presentaba un nuevo caso que compartía los problemas afásicos y comportamentales, aunque con predominio de un grave deterioro de la memoria. Este trastorno estaba causado por una neuropatología característica y diferente a los cuerpos de Pick, ampliamente distribuida por el córtex cerebral.

En las décadas posteriores, hasta la primera mitad de siglo XX, la investigación en el campo de las demencias se centró en separar la denominada enfermedad de Alzheimer (EA) de la enfermedad de Pick. En las primeras fases de la primera se describieron los síntomas amnésicos mientras que para la segunda predominó el estudio de los síntomas conductuales y del lenguaje (Leyton \& Ballard, 2016; Lillo \& Leyton, 2016).

Hacia la década de los 70, el gran auge en la investigación de la enfermedad de Alzheimer relegó a un segundo plano el interés por el estudio la enfermedad de Pick. Y no es hasta la década de los 90 cuando aparece el término de 'Demencia frontotemporal' para englobar a diferentes subtipos clínicos, que podían ser causados por varios procesos neuropatológicos además de los cuerpos de Pick. Este término resultaba, así pues, más amplio e inclusivo (Kertesz \& Munoz, 1998; Perry \& Rosen, 2016; Olney, Spina \& Miller, 2017). Por su parte, el término enfermedad de Pick se reservó para describir a los casos de DFT con neuropatología de tipo Pick (Woollacott \& Rohrer, 2016).

En este periodo, de finales del siglo pasado, dos equipos europeos (Englund, Brun, Gustafson, Passant, Mann, Neary \& Snowden, 1994) y posteriormente un equipo internacional (Neary, Snowden, Gustafson, Passant, Stuss, Black, Freedman, Kertesz, Robert, Albert, Boone, Miller, Cummings \& Benson, 1998) sentaron las bases para la clasificación diagnóstica de la DFT. 
El equipo de Neary et al. (1998) acuñó el término de Degeneración lobar frontotemporal' dividiéndolo en tres síndromes clínicos. La 'Demencia frontotemporal', fue descrita como una "profunda alteración de la personalidad y la conducta social, caracterizada por inercia y pérdida de volición social o una desinhibición social y distractibilidad, con una relativa preservación de la memoria" (Neary et al., 1998: 1546). Seguidamente, la 'Afasia progresiva no fluente' (APNF), fue presentada como:

"un trastorno del lenguaje expresivo caracterizado por una producción del habla laboriosa, con errores fonológicos y gramaticales, y dificultades para la recuperación de palabras" (Neary et al., 1998: 1546).

Por último, la 'Demencia semántica' (DS), caracterizada por un:

“deterioro severo de la nominación y un deterioro de la comprensión de las palabras que ocurre en el contexto de habla fluida, sin esfuerzo y gramaticalmente correcta" (Neary et al., 1998: 1546-1547).

Asimismo, se utiliza el término de 'DFT de variante conductual', tras la revisión de sus criterios por Rascovsky, Hodges, Knopman, Mendez, Kramer, Neuhaus, van_Swieten, Seelaar, Dopper, Onyike, Hillis, Josephs, Boeve, Kertesz, Seeley, Rankin, Johnson, Gorno-Tempini, Rosen, Prioleau-Latham, Lee, Kipps, Lillo, Piguet, Rohrer, Rossor, Warren, Fox, Galasko, Salmon, Black, Mesulam, Weintraub, Dickerson, Diehl-Schmid, Pasquier, Deramecourt, Lebert, Pijnenburg, Chow, Manes, Grafman, Cappa, Freedman, Grossman y Miller (2011), para distinguirla de las formas de DFT de variante lingüística: la 'Afasia progresiva no fluente' y la 'Demencia semántica' (Woollacott \& Rohrer, 2016).

Paralelamente, en la práctica clínica surgían dificultades para categorizar a algunos casos de EA que, lejos de presentar el inicial perfil de deterioro mnésico, debutaban con un predominio de la disfunción frontal o parietal (Mesulam, Wicklund, Johnson, Rogalski, Léger, Rademaker, Weintraub \& Bigio, 2008). Gorno-Tempini, Dronkers, Rankin, Ogar, Phengrasamy, Rosen, Johnson, Weiner y Miller (2004) describieron, por su parte, algunos casos de demencia con alteraciones en el lenguaje que no encajaban en los criterios de la DS ni la APNF.

El cambio en la conceptualización de la APP vino motivado por tres factores. En primer lugar, la división entre afasias fluentes y no fluentes, típico de la afasiología clásica, no resulta un factor diferencial tan claro en la categorización de la APP. En las variantes lingüísticas de la DFT, la reducción de la fluencia y el agramatismo pueden aparecer disociados. Por este hallazgo, surgen nuevas clasificaciones que superan el problema de la dualidad entre Demencia semántica (fluente) y Afasia progresiva primaria no fluente (Gorno-Tempini, Hillis, Weintraub, Kertesz, Mendez, Cappa, Ogar, Rohrer, Black, Boeve, Manes, Dronkers, Vandenberghe, Rascovsky, Patterson, Miller, Knopman, Hodges, Mesulam \& Grossman, 2011; Harciarek \& Kertesz, 2011; 
Thompson, Cho, Hsu, Wieneke, Rademaker, Weitner, Mesulam \& Weintraub, 2012). Como consecuencia, la correcta evaluación del agramatismo adquiere, desde entonces, mayor importancia en el diagnóstico diferencial de las APP (Mesulam et al., 2014; Thompson \& Mack, 2014).

En segundo lugar, se describe una nueva variante denominada 'Afasia progresiva logopénica' (APL) para incluir a aquellos casos que presentaban un habla lenta, laboriosa y llena de largas pausas para buscar las palabras, diferenciándose significativamente del perfil de lenguaje reductivo, más propio de la APNF (Mesulam, 1982).

Por último, se consideró que los distintos perfiles de alteración del lenguaje que se manifestaban en los pacientes con APP, tomarían la forma de una variante u otra según la localización anatómica inicial de la atrofia del córtex (Mesulam et al., 2014). De este modo, fueron surgiendo clasificaciones diagnósticas de la APP más ajustadas a los síntomas y al patrón de la enfermedad subyacente, quedando posteriormente divididas en:

a) 'APP variante no fluente o agramática' (APPvnF) estrechamente relacionada con el diagnóstico de APNF (Amici, Gorno-Tempini, Ogar, Dronkers \& Miller, 2006; Harciarek \& Kertesz, 2011; Matías-Guiu \& García-Ramos, 2013).

b) 'APP variante semántica' ( $\mathrm{APPvS})$, también conocida como 'variante temporal de la DFT', 'APP fluente' (Amici et al., 2006; Harciarek \& Kertesz, 2011; Matías-Guiu \& García-Ramos, 2013) o 'forma no agnósica de la DS' (Mesulam, 2001; Rogalski \& Mesulam, 2007; Mesulam et al., 2014).

c) 'APP variante logopénica' (APPvL), también conocida como APL (GornoTempini et al., 2004; Amici et al., 2006; Matías-Guiu \& García-Ramos, 2013), en algunas ocasiones diferenciada de otro subtipo denominado 'Afasia progresiva mixta' (Grossman, 2010) y recientemente considerado como 'espectro logopénico' (Giannini, Irwin, McMillan, Ash, Rascovsky, Wolk, van Deerlin, Lee, Trojanowski \& Grossman, 2017).

Para una revisión de la evolución histórica de la DFT y la APP consúltese el trabajo de Pérez-Lancho y García-Bercianos (2018).

\section{Criterios diagnósticos actuales}

\subsection{Criterio internacional}

Un acuerdo internacional (Gorno-Tempini et al., 2011) consolidó las bases para el diagnóstico de la APP. Una vez cumplidos los criterios generales para el diagnóstico de la APP (Tabla 1), se podría determinar la variante correspondiente (Tabla 2). 
Tabla 1. Criterios diagnósticos generales de Gorno-Tempini et al. (2011) para caracterizar la APP. ${ }^{1}$

\begin{tabular}{|c|c|}
\hline \multicolumn{2}{|r|}{ Diagnóstico de afasia progresiva primaria } \\
\hline \multirow{3}{*}{$\begin{array}{l}\text { Criterios de inclusión: } \\
3 / 3\end{array}$} & La característica clínica más prominente es la dificultad con el lenguaje. \\
\hline & Estos déficits son la principal causa de deterioro de las actividades diarias. \\
\hline & $\begin{array}{l}\text { La afasia ha de ser el principal déficit al comienzo y en las primeras fases de la } \\
\text { enfermedad. }\end{array}$ \\
\hline \multirow{4}{*}{$\begin{array}{l}\text { Criterios de exclusión: } \\
1 / 4\end{array}$} & $\begin{array}{l}\text { El patrón de los déficits se explica mejor por otro trastorno médico o no } \\
\text { degenerativo del sistema nervioso. }\end{array}$ \\
\hline & $\begin{array}{l}\text { Las alteraciones cognitivas son explicadas mejor por un diagnóstico } \\
\text { psiquiátrico. }\end{array}$ \\
\hline & $\begin{array}{l}\text { Deterioro prominente desde el inicio en memoria episódica, memoria visual o } \\
\text { visoperceptual. }\end{array}$ \\
\hline & Deterioro conductual prominente desde el inicio. \\
\hline
\end{tabular}

Tabla 2. Criterios diagnósticos específicos de Gorno-Tempini et al. (2011) para caracterizar las variantes de la afasia progresiva primaria. ${ }^{2}$

\begin{tabular}{|c|c|c|c|c|c|c|}
\hline $\begin{array}{c}\text { Afasia } \\
\text { Progresiva } \\
\text { Primaria }\end{array}$ & \multicolumn{2}{|r|}{$\begin{array}{c}\text { No fluente o agramática } \\
\text { APPvnF }\end{array}$} & \multicolumn{2}{|r|}{$\begin{array}{l}\text { Semántica } \\
\text { APPvS }\end{array}$} & \multicolumn{2}{|r|}{$\begin{array}{l}\text { Logopénica } \\
\text { APPvL }\end{array}$} \\
\hline \multirow{6}{*}{$\begin{array}{l}\text { Criterios } \\
\text { nucleares } \\
\text { Diagnóstico } \\
\text { clínico de } \\
\text { variante } \\
\text { específica de } \\
\text { APP }(2 / 2) \\
\text { Criterios } \\
\text { Específicos }\end{array}$} & \multirow[t]{2}{*}{$1 / 2$} & Agramatismo & \multirow[t]{2}{*}{$2 / 2$} & $\begin{array}{l}\text { Deterioro } \\
\text { denominación }\end{array}$ & \multirow[t]{2}{*}{$2 / 2$} & $\begin{array}{l}\text { Deterioro recuperación } \\
\text { de palabras sueltas en } \\
\text { habla espontánea y en } \\
\text { denominación } \\
\end{array}$ \\
\hline & & Apraxia del habla & & $\begin{array}{l}\text { Deterioro } \\
\text { comprensión de } \\
\text { palabras }\end{array}$ & & $\begin{array}{l}\text { Deterioro en repetición } \\
\text { de palabras y frases }\end{array}$ \\
\hline & \multirow{4}{*}{$2 / 3$} & $\begin{array}{l}\text { Deterioro comprensión } \\
\text { frases complejas }\end{array}$ & \multirow{4}{*}{$3 / 4$} & $\begin{array}{l}\text { Deterioro } \\
\text { conocimiento de } \\
\text { objetos, sobre todo } \\
\text { aquellos poco } \\
\text { familiares o poco } \\
\text { frecuentes } \\
\end{array}$ & \multirow{4}{*}{$3 / 4$} & $\begin{array}{l}\text { Errores fonológicos en } \\
\text { habla espontánea y } \\
\text { nominación }\end{array}$ \\
\hline & & $\begin{array}{l}\text { Comprensión de } \\
\text { palabras preservada }\end{array}$ & & $\begin{array}{l}\text { Dislexia superficial o } \\
\text { disgrafía }\end{array}$ & & $\begin{array}{l}\text { Conocimiento de } \\
\text { objetos y comprensión } \\
\text { de palabras conservados }\end{array}$ \\
\hline & & \multirow[t]{2}{*}{\begin{tabular}{|l}
$\begin{array}{l}\text { Conocimiento de } \\
\text { objetos preservado }\end{array}$ \\
\end{tabular}} & & \begin{tabular}{|l}
$\begin{array}{l}\text { Repetición de palabras } \\
\text { preservada }\end{array}$ \\
\end{tabular} & & \begin{tabular}{|l|} 
Producción del habla \\
preservada a nivel motor
\end{tabular} \\
\hline & & & & $\begin{array}{l}\text { Producción del habla } \\
\text { preservada (a nivel } \\
\text { gramático y motor) }\end{array}$ & & $\begin{array}{l}\text { Ausencia de } \\
\text { agramatismo franco }\end{array}$ \\
\hline \multirow[b]{2}{*}{$\begin{array}{l}\text { Diagnóstico de } \\
\text { variante de APP } \\
\text { con pruebas de } \\
\text { neuroimagen } \\
(2 / 2)\end{array}$} & \multicolumn{6}{|c|}{\begin{tabular}{l|l}
1 & Diagnóstico clínico de variante específica de APP \\
\end{tabular}} \\
\hline & $1 / 2$ & \begin{tabular}{|l} 
Atrofia (RMI) o \\
hipometabolismo / \\
hipoperfusión (SPECT) \\
en región frontal \\
posterior e insular \\
izquierdas
\end{tabular} & $1 / 2$ & $\begin{array}{l}\text { Atrofia (RMI) o } \\
\text { hipometabolismo / } \\
\text { hipoperfusión } \\
\text { (SPECT) en región } \\
\text { temporal anterior }\end{array}$ & $1 / 2$ & \begin{tabular}{|l} 
Atrofia (RMI) o \\
hipometabolismo / \\
hipoperfusión (SPECT) \\
en región perisilvana \\
posterior o parietal \\
izquierdas
\end{tabular} \\
\hline \multirow{3}{*}{\begin{tabular}{|l|} 
Diagnóstico de \\
variante de APP \\
con patología \\
definida $(2 / 2)$ \\
\end{tabular}} & \multirow{3}{*}{\begin{tabular}{|l|}
$1 / 1$ \\
$1 / 2$ \\
\end{tabular}} & \multicolumn{5}{|c|}{ Diagnóstico clínico de variante específica de APP } \\
\hline & & \multicolumn{5}{|c|}{ Evidencia histopatológica de una enfermedad neurodegenerativa } \\
\hline & & \multicolumn{5}{|c|}{ Presencia de una mutación patogénica } \\
\hline
\end{tabular}




\subsection{Criterios simplificados}

El equipo dirigido por Mesulam (Mesulam et al., 2014) propone la simplificación del diagnóstico de las variantes de APP en una aproximación bidimensional, considerando dos factores lingǘsticos nucleares: la alteración de la comprensión de palabras y la gramática de las frases.

En su propuesta, las variantes de la APP se denominaron: 'APP con agramatismo' (APP-G), 'APP semántica' (APP-S) y 'APP logopénica' (APP-L), añadiendo una cuarta categoría, 'APP mixta' (APP-M), para los casos que aún no podían clasificarse en las otras tres (ver Figura 1).

Su aportación más notable consiste en reducir la posibilidad de que un paciente tenga más de un diagnóstico, proporcionar una definición más inclusiva de la variante logopénica y reconocer el tipo de paciente con dificultades mixtas. Destacamos que, en esta clasificación, la variante logopénica ya no se caracteriza necesariamente por la alteración de la repetición, sino que se sustituye esta condición por el buen desempeño en la gramática y la comprensión. La variante gramatical ya no se hace depender de la variable fluencia.

Como principal problema, se reconoce que es un sistema de clasificación sensible a la evolución temporal de los síndromes. Pacientes en estados prodrómicos de variantes agramática o semántica podrían ser clasificados como de variante logopénica. Sin embargo, si el diagnóstico se realiza en un estadio muy avanzado de la enfermedad, los pacientes probablemente encajarán predominantemente en la categoría de 'APP mixta'. Además, la línea divisoria entre las categorías agramática y logopénica es difusa, tanto por dificultades en la evaluación de la gramática, que es difícil de cuantificar (Mesulam et al., 2014; Bisenius, Neumann \& Schroeter, 2016) como en los hallazgos de las pruebas de neuroimagen (Bisenius et al., 2016). 


\section{APP-G}

Características:

La repetición de palabras y frases y la comprensión de frases sintácticamente complejas puede estar deteriorada, pero la comprensión de palabras y frases

sencillas esta preservada.

Región con mayor atrofia:

Circunvolución frontal inferior. Patología habitual: Taupatía

\section{APP-M}

Características:

Determinado por la combinación de deterioro en la comprensión y en la gramática en fases relativamente tempranas de la enfermedad.

Región con mayor atrofia: Circunvolución frontal inferior y región anterior del lóbulo temporal.

Patología habitual:

Alzheimer

\section{APP-L}

Características:

Anomia y pausas en la recuperación de

palabras provocan pausas, parafasias

fonémicas y circunloquios. Fluencia normal en charla habitual. Dos subgrupos: CON y

SIN deterioro en la repetición. Gramática y

comprensión de palabras conservada.

Región con mayor atrofia:

Junta temporoparietal.

Patología habitual:

Alzheimer

\section{APP-S}

Características:

Gran deterioro en la comprensión y recuperación de palabras y nominación.

Gramática y repetición conservadas.

El discurso es vago y puede contener parafasias semánticas y circunloquios.

Región con mayor atrofia:

Región anterior del lóbulo temporal.

Patología habitual:

DLFT-TDP-43 tipo C

Figura 1. Clasificación diagnóstica bidimensional del criterio Mesulam et al. (2014). 3

Los criterios propuestos por Mesulam et al. (2014) que definen a la gramática y la comprensión como dimensiones nucleares en el diagnóstico diferencial de los subtipos de APP, así como la necesidad de considerar un subtipo mixto, son respaldados por el estudio de Vandenberghe (2016).

\subsection{Criterios de Botha et al. (2015)}

En 2015, el equipo americano de Botha, Duffy, Whitwell, Strand, Machulda, Schwarz, Reid, Spychalla, Senjem, Jones, Lowe, Jack y Josephs (2015) revisó de nuevo los criterios internacionales, al encontrar algunas dificultades en la aplicación de los criterios Gorno-Tempini et al. (2011). En primer lugar, una considerable proporción de pacientes no podía ser clasificada mediante los criterios de la APP o cumplían criterios de más de un subtipo. En segundo lugar, persistían las dificultades para discernir si los trastornos de la fluencia, como las pausas y vacilaciones en el discurso, deberían ser considerados como variantes agramáticas o como variantes logopénicas. Por último, pacientes con trastornos de la programación motora del habla, diagnosticados de 'Apraxia del habla' (AdH), cumplían el criterio de afasia no fluente a pesar de no cumplir los criterios nucleares para la APP (Josephs, Duffy, Strand, Machulda, Senjem, Master, Lowe, Jack Jr \& Whitwell, 2012). 
Para resolver estos problemas, Botha et al. (2015) presentaron una nueva subdivisión de las APP describiendo las siguientes entidades diagnósticas:

a) 'Apraxia progresiva del habla' (APdH), en la que la apraxia del habla es el rasgo central. En estos pacientes, el habla puede ser agramática o telegráfica, pueden mostrar dificultades en la comprensión, en la escritura y la denominación o coexistir con alteraciones neuromotrices del habla (disartria).

b) 'Afasia progresiva agramática' (APA), en la que el lenguaje verbal y/o escrito son agramáticos o telegráficos. Pueden aparecer dificultades en la comprensión verbal en la lectoescritura y en la denominación. La apraxia del habla puede estar presente, pero ha de ser de severidad igual o menor a la afasia.

c) 'Afasia progresiva logopénica' (APL), presenta una producción verbal fluente, ni agramática ni telegráfica, aunque en el discurso aparezcan pausas y vacilaciones por la dificultad para encontrar las palabras. Aparece asociada a una pobre retención auditiva de los estímulos, lo que puede afectar a la repetición y a la comprensión de frases. Las parafasias fonológicas (ej. 'pigre' por 'tigre') constituyen, en este caso, errores en la selección de los fonemas, y han de diferenciarse de las distorsiones propias de la apraxia del habla, que son debidas a un déficit en la programación de los movimientos implicados en la producción oral voluntaria.

d) 'Demencia semántica' (DS), en la que predomina una pobre comprensión de palabras y anomia. La severidad de la pérdida del significado de las palabras puede ser desproporcionada en comparación con la severidad del resto de los déficits. La producción del habla es normal en su prosodia, gramática y sintaxis, a excepción de la presencia de abundantes pausas para encontrar palabras de baja frecuencia de uso. El contenido del discurso puede ser pobre en términos de nombres y verbos, usando el paciente palabras genéricas (ej. 'la cosa', 'eso' o 'aquello'). Los errores fonológicos son raros.

e) 'Afasia progresiva fluente' (APF), es un subgrupo de las APP de variante semántica, en el que la anomia es el principal síntoma, mientras que se mantiene una buena comprensión de palabras. El habla es fluente pero no cumple criterios de APL o DS.

\section{Caracterización de las APP}

Partiendo de los criterios que hemos presentado hasta aquí, esto es, el estudio internacional de Gorno-Tempini et al. (2011), la aproximación simplificada de Mesulam et al. (2014) y la clasificación de Botha et al. (2015) vamos a agrupar las características principales del lenguaje de las variantes de APP, en sus tres formas clásicas. Además, presentamos algunos estudios de neuroimagen que describen las correspondencias anatomo-funcionales de cada una de ellas. 


\subsection{La Afasia progresiva primaria agramática}

Se produce por una degeneración del opérculo frontal izquierdo y la ínsula rostral, con atrofia adicional de los ganglios basales izquierdos, aunque en algunos casos esta atrofia sea muy sutil o no aparezca (Sajjadi, Sheickh-Bahaei, Cross, Guillard, Scoffings \& Nestor, 2017).

Los déficits de denominación están ausentes o son escasos y no se ha encontrado asociación con atrofia de ninguna de las regiones anatómicas encargadas de la integración léxico-semántica (Migliaccio, Boutet, Valabregue, Ferrieux, Nogues, Lehéricy, Dormont, Levy, Dubois \& Teichmann, 2016). Sin embargo, a nivel gramatical, estos pacientes presentan menor fluencia, menos expresiones gramaticales, más expresiones no verbales, más errores sintácticos y semánticos, y menos expresiones complejas que en las restantes variantes, errores relacionados con alteraciones anatómicas en el área de Broca (Tetzloff, Utianski, Duffy, Clark, Strand, Josephs \& Whitwell, 2018).

Aunque la mayoría de estos pacientes presenten puntuaciones bajas en fluidez, esta característica puede compartirse con la variante logopénica. Es preciso destacar, por tanto, que el rasgo esencial es el agramatismo y que este debe ser disociado de la fluencia (Thompson et al., 2012). Los individuos con afasia progresiva primaria agramática presentan patrones específicos de déficit agramático, distintos de los del resto de las variantes, pero similares al agramatismo propio de las afasias motoras producidas por accidentes vasculares cerebrales, con las que comparten las regiones anatómicas afectadas (Thompson \& Mack, 2014).

Así pues, la afasia progresiva primaria agramática se caracterizaría por presencia de anormalidades en el discurso, siendo este trabajoso, lento y telegráfico, con reducción de la sintaxis. Su curso de asocia tanto con trastornos neuromotrices (disartria) como con problemas de programación del habla (apraxia del habla). Por ello, son frecuentes los errores parafásicos tanto de tipo fonético (cambio de rasgos de los fonemas por modificaciones en los puntos de articulación) como fonológicos (intercambio de fonemas sin relación entre sî), respectivamente.

La comprensión del lenguaje no afecta a las palabras aisladas sino a las frases sintácticamente complejas, mientras que el conocimiento de objetos está conservado. Se altera la capacidad de repetición debido a las dificultades de fluencia y la denominación es siempre defectuosa, afectando más a los verbos que a los nombres. La lectura y la escritura se alteran según un perfil fonológico. Esto se refleja, respectivamente, en paralexias fonológicas (errores de sustitución de fonemas al leer) y paragrafias fonológicas (sustitución de grafemas al escribir). Además, aparecen mayores dificultades para deletrear pseudopalabras. Esta variante incluiría la APdH y la APA de la clasificación de Botha et al. (2015). 


\subsection{La Afasia progresiva primaria semántica}

Se caracteriza por una pérdida del conocimiento amodal con una reducción de vocabulario expresivo y de la comprensión de palabras. Estudios radiológicos destacan la atrofia predominante del lóbulo temporal anterior izquierdo, resultando este hallazgo un sólido indicador diagnóstico (Sajjadi et al., 2017).

El paciente presenta un discurso fluente pero vacío de contenido, empleando palabras genéricas para compensar el déficit de acceso a los nombres. Los mecanismos dañados afectan fundamentalmente a la comprensión de palabras, es decir al procesamiento léxico-semántico, que en estudios de morfometría basada en vóxel correlaciona con alteraciones en las porciones superiores del polo temporal izquierdo. Por otra parte, se ha localizado una zona de solapamiento entre la zona superior e intermedia del lóbulo temporal izquierdo que procesaría bidireccionalmente la información de semántica a léxica y de léxica a semántica (Migliaccio et al., 2016). Este hallazgo se correspondería con la asociación de las alteraciones semánticas ya descritas con ciertos problemas en la recuperación de la forma fonológica de las palabras (Sanches, Routier, Colliot \& Teichmann, 2018).

La afasia progresiva primaria semántica no cursa con alteraciones neuromotrices del habla ni apraxia, por lo que los errores parafásicos típicos son de carácter verbalsemántico (ej. 'león' por 'tigre') y no fonológico. Los pacientes pueden preguntar, en la conversación, por el significado de palabras que no entienden, pero también pueden presentar problemas para elegir qué imagen se relaciona con una palabra.

La repetición está preservada y, sin embargo, las palabras de menor frecuencia no se pueden definir. Aparecen errores en la lectura y escritura de palabras irregulares, en un perfil análogo al de la alexia y agrafia de tipo superficial o léxica, unido a una afectación del componente ortográfico (Pulvemüller, Cooper-Pye, Dine, Houk, Nestor \& Patterson, 2010; Sanches et al., 2018). Tempranamente, se detectan problemas con la denominación de palabras poco frecuentes, conservándose el conocimiento de las palabras familiares hasta bien avanzada la enfermedad. La anomia afecta más a los nombres que a los verbos.

La memoria episódica y de trabajo están preservadas y, sin embargo, se hace notorio el deterioro pragmático originado por las dificultades específicas para inferir el significado del contexto. La información visual y otros dominios no verbales se afectarían tardíamente, apareciendo prosopagnosia y agnosia visual. Esta variante comprendería a la DS y a la APF de la clasificación de Botha et al. (2015).

\subsection{La 'Afasia progresiva primaria logopénica'}

Está altamente asociada con la patología de la enfermedad de Alzheimer y concuerda con hallazgos de atrofia perisilviana posterior izquierda. Sin embargo, la 
ausencia de atrofia no excluye el diagnóstico, apoyando la idea de que hay dificultades para distinguir radiológicamente los patrones neuroanatómicos tanto de la variante logopénica como de la agramática (Sajjadi et al., 2017).

Estudios recientes plantean la existencia de dos subtipos de APP logopénica. El primero compartiría los biomarcadores positivos de amiloide y un patrón de atrofia cortical similar al de la Enfermedad de Alzheimer. El segundo grupo lo constituirían los pacientes amiloide-negativos, con un patrón metabólico cerebral diferente y una atrofia cortical claramente asimétrica y de predominio izquierdo (Matías-Guiu, Cabrera-Martín, Moreno-Ramos, Valles-Salgado, Fernández-Matarrubia, Carreras \& Matías-Guiu, 2015; Whitwell, Duffy, Strand, Machulda, Senjem, Schwarz, Reid, Baker, Pekerson, Lowe, Rademakers, Jack Jr \& Josephs, 2015).

El lenguaje en la variante logopénica se caracterizaría por presentar frecuentes pausas en el discurso y circunloquios originados por la dificultad del paciente para encontrar las palabras, manteniendo conservado el acceso al significado de las mismas y la estructura gramatical de las frases. Esta dificultad para encontrar las palabras tiende a elicitar parafasias de tipo fonológico. Las alteraciones en las regiones de la porción temporal posterior izquierda son responsables del deterioro del procesamiento semántico-léxico, lo que dificulta a los enfermos el acceso a la forma de las palabras que se han de denominar, aunque comprendan su significado (Migliaccio et al., 2016). En contraste, los componentes léxico-semánticos y sintácticos están relativamente preservados en las primeras fases de la enfermedad (Sanches et al., 2018).

La repetición de frases puede estar más deteriorada que la de palabras, por incapacidad de mantener activado el material verbal en la memoria de trabajo. La lectura y la escritura se alteran según un perfil de alexia y agrafia fonológicas. En las primeras fases de la enfermedad, no se dan alteraciones pragmáticas, pero en este caso se correspondería con la APL de Botha et al. (2015).

A continuación, presentamos las características del deterioro del lenguaje en las tres variantes principales de la APP (Tabla 3) considerando las aportaciones de Harciarek y Kertesz (2011), Duffy, Strand y Josephs (2014), Mesulam (2016) y Poole, Brodtmann, Darby y Vogel (2017). 
Tabla 3. Características principales de los subtipos de 'Afasia progresiva primaria'.4

\begin{tabular}{|c|c|c|c|}
\hline Características & APP no fluente & APP Logopénica & APP Semántica \\
\hline Habla & $\begin{array}{l}\text { Disfluente, costosa, } \\
\text { agramática, telegráfica, } \\
\text { con pausas frecuentes } \\
\text { por fallo en la búsqueda } \\
\text { de palabras }\end{array}$ & $\begin{array}{l}\text { Lenta, logopénica, con } \\
\text { pausas, vacilaciones y } \\
\text { circunloquios debido al } \\
\text { fallo en la búsqueda de } \\
\text { palabras }\end{array}$ & $\begin{array}{l}\text { Fluente, gramaticalmente } \\
\text { correcta pero con } \\
\text { circunloquios, } \\
\text { perseveraciones temáticas } \\
\text { y jerga semántica } \\
\end{array}$ \\
\hline Parafasias & Fonémicas o Fonéticasb & Fonémicas & Semánticas \\
\hline Apraxia del habla & $\mathrm{Si}$ & No & No \\
\hline Disartria & Posible & Raramente & No \\
\hline $\begin{array}{l}\text { Comprensión de } \\
\text { palabas }\end{array}$ & Preservada & Preservada & Deteriorada \\
\hline $\begin{array}{l}\text { Comprensión de } \\
\text { frases }\end{array}$ & $\begin{array}{l}\text { Deteriorada, si la frase es } \\
\text { sintácticamente compleja }\end{array}$ & $\begin{array}{l}\text { Deteriorada para frases } \\
\text { largas a }\end{array}$ & Deteriorada \\
\hline Repetición de palabras & Deteriorada & Preservada & Preservada \\
\hline Repetición de frases & Deteriorada & $\begin{array}{l}\text { Puede estar deteriorada } \\
\text { para frases largas a }\end{array}$ & Preservada \\
\hline Denominación & Deteriorada & Deteriorada & Deteriorada \\
\hline Sintaxis & Reducida & Inicialmente preservada & Preservada \\
\hline Gramática & Deteriorada & Preservada & Preservada \\
\hline $\begin{array}{l}\text { Pragmática del } \\
\text { lenguaje }\end{array}$ & Preservada & Preservada & Deterioro severo \\
\hline Lectura y ortografía & $\begin{array}{l}\text { Dificultad para leer } \\
\text { pseudopalabras. Agrafia } \\
\text { fonológica }\end{array}$ & $\begin{array}{l}\text { Dificultad para leer } \\
\text { pseudopalabras. }\end{array}$ & $\begin{array}{l}\text { Dificultad para leer } \\
\text { palabras irregulares. } \\
\text { Agrafia léxica }\end{array}$ \\
\hline Memoria episódica & Relativamente preservada & $\begin{array}{l}\text { Deterioro en etapas } \\
\text { avanzadas de la } \\
\text { enfermedad }\end{array}$ & Relativamente preservada \\
\hline Memoria de trabajo & Puede estar afectada & $\begin{array}{l}\text { Puede estar severamente } \\
\text { afectada a }\end{array}$ & Típicamente preservada \\
\hline Principal déficit & $\begin{array}{l}\text { Formulación del lenguaje } \\
\text { o de la programación } \\
\text { motora } \\
\end{array}$ & $\begin{array}{l}\text { Denominación y } \\
\text { encontrar palabras } \\
\text { (repetición a) }\end{array}$ & Significado del mensaje \\
\hline
\end{tabular}

\section{CONCLUSIONES}

La APP es una entidad heterogénea que incluye síndromes clínicos que se pueden solapar. A pesar de ello, la comparación de los perfiles lingüísticos, junto a los estudios de neuroimagen, deberían permitirnos establecer un diagnóstico diferencial cada vez más preciso y precoz. Al tratarse de enfermedades neurodegenerativas, es importante determinar cuáles son los procesos del lenguaje afectados en los estadios iniciales, antes de que el deterioro afecte a múltiples dominios cognitivos (Pérez-Lancho \& García-Bercianos, 2018).

Actualmente, contamos con tres versiones de criterios diagnósticos de las APP (Gorno-Tempini et al., 2011; Mesulam et al., 2014; Botha et al., 2015) que ofrecen una caracterización lingüística de las patologías cada vez más completa. Parece que la variante semántica es la que ha sido mejor descrita y la que presenta un perfil clínico más estable en todas las clasificaciones, incluyendo hallazgos neurorradiológicos de 
claro valor diagnóstico (Sajjadi et al., 2017). En esta línea, un reciente estudio muestra que la casuística de APP se distribuye en dos grupos. El primero, formado por aquellos pacientes con 'APP semántica', con marcados déficits en el procesamiento semántico, tanto verbal como no verbal. El segundo, formado por aquellos pacientes con déficits en el discurso, la repetición y la gramática, que incluye a casos tanto de 'APP no fluente', como de 'APP logopénica' y algunos de 'APP mixta' (Hoffman, Sajjadi, Patterson \& Nestor, 2017).

Como vemos, persiste la controversia sobre la categorización de la 'APP logopénica', cuyas fronteras diagnósticas están aún definiéndose (Matías-Guiu et al, 2015; Whitwell et al., 2015). Esta dificultad podría resolverse, en primer lugar, excluyéndola del espectro DFT, para considerar a los casos con hallazgos positivos de beta-amiloide como una forma lingüística de la EA. Aunque la APP-L y la EA comparten mayoritariamente la neuropatología subyacente, algunos autores sostienen que ninguna medida del lenguaje puede ser definitiva para el diagnóstico diferencial, con lo que la APP-L sería un subtipo de EA (Rogalski, Sridhar, Rader, Martersteck, Chen, Cobia \& Mesulam, 2016). Por otro lado, algunos autores defienden que las diferencias entre ambas entidades APP-L y EA son significativas cuando los perfiles de atrofia de la APP-L son asimétricos y de predominio izquierdo (Whitwell et al., 2015). Otros autores refieren que el solapamiento entre ambos puede provenir de dificultades en la evaluación de la memoria episódica verbal. Esta memoria, deteriorada en la EA, en los casos de APP-L sería una manifestación de las dificultades de recuperación léxica y no de alteraciones mnésicas propiamente dichas (Win, Pluta, Yushkevich, Irwin, McMillan, Rascovsky, Wolk \& Grossman, 2017). Finalmente, el equipo de Giannini et al. (2017) propone utilizar el concepto de 'espectro logopénico', para englobar a tres subsíndromes diferentes, en función del cumplimiento de los criterios diagnósticos nucleares y específicos propuestos por Gorno-Tempini et al. (2011).

Otra consideración importante a reseñar la es la necesidad de evaluar correctamente los trastornos agramáticos, nucleares de la variante agramática de la APP. Evaluar los componentes morfosintácticos a través del análisis del discurso oral incluye el efecto modulador de otras variables como la disfunción ejecutiva o la ansiedad social al hablar, cuyo impacto no ha sido tenido en cuenta hasta ahora en la investigación, y por lo tanto podría ser sustituido por tareas de análisis gramatical del lenguaje escrito (Tetzloff et al., 2018). Este es un aspecto que debería ser considerado con más detalle en el futuro.

Por otra parte, también queremos destacar que las variantes lingüísticas de la DFT presentan un alto grado de solapamiento con síndromes clínicos de tipo motor, entre los que se incluyen el 'Parkinsonismo atípico' y la 'Esclerosis lateral amiotrófica', llegando algunos autores a hablar de un continuo cuyos extremos serían la 'Esclerosis lateral amiotrófica’ y la DFT (Pérez-Lancho \& García-Bercianos, 2018). Respecto a las 
disfunciones motoras del habla, destacamos el trabajo de Poole et al. (2017), un metaanálisis que pone en relieve la cantidad de variables que entran en juego en la evaluación de dichas disfunciones a través de las diferentes formas de DFT, APP y AdH, su importancia en la clasificación diagnóstica y las líneas futuras de la investigación en este ámbito.

En general, la DFT es una enfermedad de largo recorrido con una supervivencia de entre 6 y 12 años, similar a la de la EA y con una edad de inicio más temprana. Sin embargo, en aquellos casos en los que la DFT que se asocia con 'Esclerosis lateral amiotrófica', se dispara la mortalidad prematura y se reduce enormemente la esperanza de vida (Kansal, Mareddy, Sloane, Minc, Rabins, Mc Gready \& Onyike, 2016). Este hecho origina nuevas dificultades clínicas a la hora realizar el diagnóstico, ya que hay que considerar la probabilidad de que un paciente con un trastorno primario predominantemente motor pueda evolucionar hacia una forma de APP y viceversa. Por ello, en este trabajo hemos resaltado las clasificaciones que analizan las formas de apraxia del habla y la disartria de los enfermos con APP (Botha et al., 2015).

Como conclusión final, aunque la discusión sobre el tema permanece abierta, la importancia de conocer y evaluar las características del lenguaje y sus correlatos neuroanatómicos en las diferentes variantes de la APP es doble: en primer lugar, para favorecer la identificación precoz de estos trastornos y, en segundo lugar, para permitir el diseño de programas de intervención cognitivo-lingüística más ajustados a cada perfil, e implementarlos desde las primeras fases evolutivas de la enfermedad.

\section{REFERENCIAS BIBLIOGRÁFICAS}

Alzheimer, A. (1911). Über eigenartige Krankheitsfälle des späteren Alters Zeitschrift Für Die Gesamte. Neurologie Und Psychiatrie, 4, 356-385.

Amici, S., Gorno-Tempini, M. L., Ogar, J. M., Dronkers, N. F. \& Miller, B. L. (2006). An overview on primary progressive aphasia and its variants. Behavioural Neurology, 17(2), 77-87.

Bisenius, S., Neumann, J. \& Schroeter, M. L. (2016). Validating new diagnostic imaging criteria for primary progressive aphasia via anatomical likelihood estimation meta-analyses. European Journal of Neurology, 23(4), 704-712.

Botha, H., Duffy, J. R., Whitwell, J. L., Strand, E. A., Machulda, M. M., Schwarz, C. G., Reid, R. I., Spychalla, A. J., Senjem, N. L., Jones, D. T., Lowe, V., Jack, C. R. \& Josephs, K. A. (2015). Classification and clinicoradiologic features of primary progressive aphasia (PPA) and apraxia of speech. Cortex, 69, 220-236.

Duffy, J. R., Strand, E.A. \& Josephs, K. A. (2014). Motor speech disorders associated with primary progressive aphasia, Aphasiology, 28(8-9), 1004-1017. 
Englund, B., Brun, A., Gustafson, L., Passant, U., Mann, D., Neary, D. \& Snowden, J. S. (1994). Clinical and neuropathological criteria for frontotemporal dementia. Journal of Neurology, Neurosurgery and Psychiatry, 57(4), 416-8.

Giannini, L. A. A., Irwin, D. J., McMillan, C. T., Ash, S., Rascovsky, K., Wolk, D. A., Van Deerlin, V. M., Lee, E. B., Trojanowski, J. Q. \& Grossman, M. (2017). Clinical marker for Alzheimer disease pathology in logopenic primary progressive aphasia. Neurology, 88(24), 2276-2284.

Gorno-Tempini, M. L., Dronkers, N. F., Rankin, K. P., Ogar, J. M., Phengrasamy, L., Rosen, H. J., Johnson, J. K., Weiner, M. W. \& Miller, B. L. (2004). Cognition and anatomy in three variants of primary progressive aphasia. Annals of Neurology, 55(3), 335-346.

Gorno-Tempini, M. L., Hillis, A. E., Weintraub, S., Kertesz, A., Mendez, M., Cappa, S. F., Ogar, J. M., Rohrer, J. D., Black, S., Boeve, B. F., Manes, F., Dronkers, N. F., Vandenberghe, R., Rascovsky, K., Patterson, K., Miller, B. L., Knopman, D. S., Hodges, J. R., Mesulam, M. M. \& Grossman, M. (2011). Classification of primary progressive aphasia and its variants. Neurology, 76(11), 1006-1014.

Grossman, M. (2010). Primary progressive aphasia: Clinicopathological correlations. Nature Reviews Neurology, 6(2), 88-97.

Harciarek, M. \& Kertesz, A. (2011). Primary progressive aphasias and their contribution to the contemporary knowledge about the brain-language relationship. Neuropsychology Review, 21(3), 271-287.

Hoffman, P., Sajjadi, S. A., Patterson, K. \& Nestor, P. J. (2017). Data-driven classification of patients with primary progressive aphasia. Brain and Language, $174,86-93$.

Josephs, K. A., Duffy, J. R., Strand, E. A., Machulda, M. M., Senjem, M. L., Master, A. V., Lowe, V. J., Jack Jr, C. R. \& Whitwell, J. L. (2012). Characterizing a neurodegenerative syndrome: Primary progressive apraxia of speech. Brain, 135(5), 1522-1536.

Kansal, K., Mareddy, M., Sloane, K. L., Minc, A. A., Rabins, P. V., Mc Gready, J. B. \& Onyike, C. U. (2016). Survival in frontotemporal dementia phenotypes: A meta-analysis. Dementia and geriatric cognitive disorders, 41(1-2), 109-122.

Kertesz, A. \& Munoz, D. (1998). Pick's disease, frontotemporal dementia, and Pick complex: Emerging concepts. Archives of Neurology, 55(3), 302-304.

Leyton, C. E. \& Ballard, K. J. (2016). Primary progressive aphasia: Conceptual evolution and challenges. Neuroscience and Neuroeconomics, 5, 9-18. 
Lillo, P. \& Leyton, C. (2016). Demencia frontotemporal, cómo ha resurgido su diagnóstico. Revista Médica Clínica Las Condes, 27(3), 309-318.

Matías-Guiu, J. A. \& García-Ramos, R. (2013). Afasia progresiva primaria: Del síndrome a la enfermedad. Neurología, 28(6), 366-374.

Matías-Guiu, J. A., Cabrera-Martín, M. N., Moreno-Ramos, T., Valles-Salgado, M., Fernandez-Matarrubia, M., Carreras, J. L. \& Matías-Guiu, J. (2015). Amyloid and FDG-PET study of logopenic primary progressive aphasia: Evidence for the existence of two subtypes. Journal of Neurology, 262(6), 1463-1472.

Mesulam, M. M. (1982). Slowly progressive aphasia without generalized dementia. Annals of Neurology, 11(6), 592-598.

Mesulam, M. M. (2001). Primary progressive aphasia. Annals of Neurology, 49(4), 425432.

Mesulam, M. M. (2016). Primary Progressive Aphasia and the Left Hemisphere Language Network. Dementia and Neurocognitive Disorders, 15(4), 93-102.

Mesulam, M. M., Rogalski, E. J., Wieneke, C., Hurley, R. S., Geula, C., Bigio, E. H., Thompson, C. K. \& Weintraub, S. (2014). Primary progressive aphasia and the evolving neurology of the language network. Nature Reviews Neurology, 10(10), 554-569.

Mesulam, M. M., Wicklund, A., Johnson, N., Rogalski, E., Léger, G. C., Rademaker, A., Weintraub, S. \& Bigio, E. H. (2008). Alzheimer and frontotemporal pathology in subsets of primary progressive aphasia. Annals of Neurology, 63(6), 709-719.

Migliaccio, R., Boutet, C., Valabregue, R., Ferrieux, S., Nogues, M., Lehéricy, S., Dormont, D., Levy, R., Dubois, B. \& Teichmann, M. (2016). The brain network of naming: A lesson from primary progressive aphasia. PloS one, 11(2).

Neary, D., Snowden, J. S., Gustafson, L., Passant, U., Stuss, D., Black, S., Freedman, M., Kertesz, A., Robert, P. H., Albert, M., Boone, K., Miller, B. L., Cummings, J. \& Benson, D.F. (1998). Frontotemporal lobar degeneration: A consensus on clinical diagnostic criteria. Neurology, 51(6), 1546-1554.

Olney, N. T., Spina, S. \& Miller, B. L. (2017). Frontotemporal dementia. Neurologic Clinics, 35(2), 339-374.

Pérez-Lancho, M. C. \& García-Bercianos, S. (2018). Demencia frontotemporal: Fronteras diagnósticas. International Journal of Developmental and Educational Psychology, 4(1), 67-76. 
Perry, D. C. \& Rosen, H. J. (2016). Frontotemporal dementia. En M. D. Geschwind \& C. R. Belkoura (Eds.), Non-Alzheimer's and Atypical Dementia (pp. 49-63). Oxford: John Wiley \& Sons.

Pick, A. (1904). Zur Symptomatologie der linksseitigen Schläfenlappenatrophie. Monatschrift für Psychiatrie und Neurologie, 16, 378-88.

Pievani, M., Filippini, N., Van Den Heuvel, M. P., Cappa, S. F. \& Frisoni, G. B. (2014). Brain connectivity in neurodegenerative diseases from phenotype to proteinopathy. Nature Reviews: Neurology, 10(11), 620-33.

Poole, M. L., Brodtmann, A., Darby, D. \& Vogel, A. P. (2017). Motor Speech Phenotypes of Frontotemporal Dementia, Primary Progressive Aphasia, and Progressive Apraxia of Speech. Journal of Speech, Language, and Hearing Research, 60(4), 897-911.

Pulvemüller, F., Cooper-Pye, E., Dine, C., Hauk, O., Nestor, P. J. \& Patterson, K. (2010). The word processing deficit in Semantic Dementia: All categories are equal, but some categories are more equal than others. Journal of Cognitive Neuroscience, 22, 2027-2041.

Rascovsky, K., Hodges, J. R., Knopman, D., Mendez, M. F., Kramer, J. H., Neuhaus, J., van_Swieten, J. C., Seelaar, H., Dopper, E. G. P., Onyike, C. U., Hillis, A. E., Josephs, K. A., Boeve, B. F., Kertesz, A., Seeley, W. W., Rankin, K. P., Johnson, J. K., Gorno-Tempini, M., Rosen, H., Prioleau-Latham, C. E., Lee, A., Kipps, C. M., Lillo, P., Piguet, O., Rohrer, J. D., Rossor, M. N., Warren, J. D., Fox, N. C., Galasko, D., Salmon, D. P., Black, S. E., Mesulam, M., Weintraub, S., Dickerson, B. C., Diehl-Schmid, J., Pasquier, F., Deramecourt, V., Lebert, F., Pijnenburg, Y., Chow, T. W., Manes, F., Grafman, J., Cappa, S. F., Freedman, M., Grossman, M. \& Miller, B. L. (2011). Sensitivity of revised diagnostic criteria for the behavioural variant of frontotemporal dementia. Brain, 134(9), 2456-2477.

Ratnavalli, E., Brayne, C., Dawson, K. \& Hodges, J.R. (2002). The prevalence of frontotemporal dementia. Neurology, 58(11), 1615-1621.

Rogalski, E. \& Mesulam, M. M. (2007). An update on primary progressive aphasia. Current Neurology and Neuroscience Reports, 7(5), 388-392.

Rogalski, E., Sridhar, J., Rader, B., Martersteck, A., Chen, K., Cobia, D., Thompson, C. K., Weintraub, S., Bigio, E. H. \& Mesulam, M. M. (2016). Aphasic variant of Alzheimer disease Clinical, anatomic, and genetic features. Neurology, 87(13), 1337-1343. 
Sajjadi, S. A., Sheikh-Bahaei, N., Cross, J., Gillard, J. H., Scoffings, D. \& Nestor, P. J. (2017). Can MRI visual assessment differentiate the variants of primaryprogressive aphasia? American Journal of Neuroradiology, 38(5), 954-960.

Sanches, C., Routier, A., Colliot, O. \& Teichmann, M. (2018). The structure of the mental lexicon: What primary progressive aphasias reveal. Neuropsychologia, 109, 107-115.

Tetzloff, K. A., Utianski, R. L., Duffy, J. R., Clark, H. M., Strand, E. A., Josephs, K. A. \& Whitwell, J. L. (2018). Quantitative analysis of agrammatism in agrammatic primary progressive aphasia and dominant apraxia of speech. Journal of Speech, Language, and Hearing Research, 61(9), 2337-2346.

Thompson, C. K. \& Mack, J. E. (2014). Grammatical impairments in PPA. Aphasiology, 28(8-9), 1018-1037.

Thompson, C. K., Cho, S., Hsu, C. J., Wieneke, C., Rademaker, A., Weitner, B. B., Mesulam, M. M. \& Weintraub, S. (2012). Dissociations between fluency and agrammatism in primary progressive aphasia. Aphasiology, 26(1), 20-43.

Toribio-Díaz, M. E. \& Morera-Guitart, J. (2008). Clasificación clínica y biomolecular de las demencias frontotemporales. Rev Neurol, 47, 588-98.

Vandenberghe, R. (2016). Classification of the primary progressive aphasias: Principles and review of progress since 2011. Alzheimer's Research \& Therapy, 8(1), 16.

Whitwell, J. L., Duffy, J. R., Strand, E. A. Machulda, M. M., Senjem, M. L., Schwarz, C. G., Reid, R., Baker, M. C., Pekerson, R. B., Lowe, V. J., Rademakers, R., Jack Jr., C. R. \& Josephs, K. A. (2015). Clinical and neuroimaging biomarkers of amyloid-negative logopenic primary progressive aphasia. Brain Lang, 142, 45-53.

Win, K. T., Pluta, J., Yushkevich, P., Irwin, D. J., McMillan, C. T., Rascovsky, K., Wolk, D. \& Grossman, M. (2017). Neural correlates of verbal episodic memory and lexical retrieval in Logopenic Variant Primary Progressive Aphasia. Frontiers in Neuroscience, 11,330.

Woollacott, I. O. \& Rohrer, J. D. (2016). The clinical spectrum of sporadic and familial forms of frontotemporal dementia. Journal of Neurochemistry, 138(S1), 6-31.

\section{NOTAS}

${ }^{1} \mathrm{x} / \mathrm{y}$ : Han de cumplirse $\mathrm{x}$ de y criterios. Adaptado de: 'Classification of primary progressive aphasia and its variants' por Gorno-Tempini et al., 2011, Neurology, 76(11), 1006-1014. 
$2 \mathrm{x} / \mathrm{y}$ : Han de cumplirse $\mathrm{x}$ de y criterios. SPECT: Tomografía computarizada de emisión monofotónica. RMI: Resonancia Magnética por Imagen. Adaptado de: 'Classification of primary progressive aphasia and its variants’ por Gorno-Tempini et al., 2011, Neurology, 76(11), 1006-1014. Las regiones cerebrales implicadas en cada uno de los subtipos de APP que aparecen en esta clasificación se han visto confirmadas por trabajos posteriores, sintetizados en el metanálisis de Bisenius, Neumann y Schroeter (2016).

3 Adaptado de 'Primary progressive aphasia and the evolving neurology of the language network' por Mesulam et al., 2014, Nature Reviews Neurology, 10(10), 554-569.

${ }^{4}$ a) Dependiendo del criterio empleado, si $\mathrm{APPvL}$ con deterioro en la repetición (Criterio Gorno-Tempini et al., 2011) o el de APP-L (Mesulam et al., 2014) según el cual puede haber, o no, deterioro en la repetición. b) Dependiendo de si se trata de apraxia del habla progresiva. 